\title{
Propuesta de Flipped Classroom para el desarrollo de las competencias genéricas en estudiantes de ingeniería
}

\author{
Agustín Lagunes Dominguez \\ Universidad Veracruzana \\ aglagunes@uv.mx
}

\author{
Luis Alberto Tafur Jiménez \\ Universidad de San Buenaventura \\ decano.ingenieria@usbmed.edu.co
}

\author{
Julián Darío Giraldo Ocampo \\ Universidad de San Buenaventura \\ ingenieria.sistemas@usbmed.edu.co
}

(Tipo de Artículo: Investigación Científica y Tecnologica. Recibido el 15/12/2017. Aprobado el 15/02/2017)

\begin{abstract}
Resumen. Este artículo trata sobre la importancia de las competencias en los estudiantes de pregrado, define las competencias, muestra sus ventajas, indica su clasificación y, sobre todo, describe las principales competencias genéricas deseables según la Agencia Nacional de Evaluación de la Calidad y Acreditación (ANECA). Posterior a esto, se trata el Flipped Classroom, su definición, sus principios, algunas recomendaciones para su implementación y se muestran sus tres principales ventajas. El principal aporte de este trabajo es una propuesta que tiene como base al Flipped Classroom y como pilares a las actividades preclase y las actividades en clase, donde estas acciones deben tener una fuerte conexión que les permita trabajar coordinadamente, integrado en la planeación estratégica didáctica para desarrollar las competencias. Dentro de las principales conclusiones se tienen es que el Flipped Classroom es ideal para desarrollar las competencias genéricas deseables, tanto las instrumentales, personales y las sistemáticas. Otra conclusión es que no hay modelo pedagógico o propuesta didáctica que tenga éxito sin el trabajo arduo del profesor.
\end{abstract}

Palabras clave. Competencias, Estudiantes, Ingeniería, Flipped Classroom, Pregrado

\section{Flipped Classroom proposal for the development of generic competencies in engineering students}

\begin{abstract}
This article discusses the importance of competencies in engineering students, defining competencies, showing their advantages, indicating their classification and, above all, describing the main generic competencies according to the National Agency for Quality Assessment and Accreditation (ANECA, for its acronym in Spanish). Afterwards, the Flipped Classroom is discussed, its definition, its principles, some recommendations for its implementation and its three main advantages are described. The main contribution of this work is given by a proposal based on the Flipped Classroom, having as pillars the pre and in class activities, where these actions must have a strong connection that allows them to work in a coordinated way, integrated in a didactic strategic planning to develop competencies. The conclusions indicate that the Flipped Classroom is ideal for the development of desirable generic competencies, such as instrumental, personal and systematic. Other conclusion points out that there is no pedagogical model or didactic proposal successful that is successful without the hard work of the teacher.
\end{abstract}

Keywords. Competencies, Students, Engineering, Flipped Classroom, Undergraduate. 
Ingenierías USBMed, Vol. 8, No. 1, Enero-Junio 2017

\section{Nomenclatura}

ANECA: Agencia Nacional de Evaluación de la Calidad y Acreditación.

EEES: Espacio Europeo de Educación Superior.

OCDE: Organización para la Cooperación y el Desarrollo Económicos

\section{Introducción}

Este artículo es una propuesta para desarrollar las competencias genéricas establecidas por la ANECA en el marco del EEES, para ello, se hace un recorrido por el concepto de competencias, sus ventajas, los tipos de competencias, terminando este apartado de la introducción con las competencias genéricas establecidas por ANECA para todos los pregrados.

Como segundo aspecto de la introducción se define lo que es Flipped Classroom, sus principios y algunas recomendaciones para implementarlas.

\subsection{Las competencias}

Dentro de las dos definiciones que tiene la Real Academia Española para competencia, la segunda es la más apropiada para esta investigación la cual indica que es la Pericia, aptitud, idoneidad para hacer algo o intervenir en un asunto determinado [1].

Por otro lado, para Van-der Hofstadt y Gómez Gras [2] competencia es un conjunto identificable y evaluable de conocimientos, actitudes, valores y habilidades relacionados entre sí que permiten desempeños satisfactorios en situaciones reales de trabajo, según estándares utilizados en el área ocupacional.

Una tercera definición de competencia es la del proyecto Tuning que la define como una combinación dinámica de atributos en relación a conocimientos, habilidades, actitudes y responsabilidades que describen los resultados de aprendizaje que han de ser alcanzados en un programa educativo y el grado de suficiencia con que una persona es capaz de desempeñarlos [3].

Así mismo la OCDE define la competencia como la capacidad para responder a demandas y llevar al cabo tareas de forma adecuada. Además, indica que las competencias están compuestas por habilidades cognitivas y prácticas, conocimientos, motivación, valores, actitudes, emociones y otros componentes sociales y conductuales [4].

Como se aprecia en todas estas definiciones, una competencia incluye los conocimientos, las habilidades y los valores para poder desarrollar una actividad en el mundo profesional y con este enfoque se pretende reducir la brecha que existe entre la universidad y el campo profesional.

\subsubsection{Ventajas de las competencias}

Trabajar en educación en un enfoque basado en competencias tiene una serie de ventajas, algunas de ellas fueron mencionadas en el informe final del Tuning
Educational Structures in Europe de González y Wagenaar [3], las cuales se comentan a continuación.

a) Fomenta la transparencia en los perfiles profesionales y académicos de las titulaciones $y$ programas de estudio y favorece un énfasis cada vez mayor en los resultados.

Esta ventaja se refiere a la transparencia de la competencia, que, al definir una competencia para una experiencia educativa o un programa educativo, sea conocido por todos y por lo tanto, tener la idea clara del profesionista que tendrán.

b) Desarrollo del nuevo paradigma de educación primordialmente centrada en el estudiante y la necesidad de encauzarse hacia la gestión del conocimiento.

Bajo el enfoque por competencias el estudiante adquiere importancia y las tareas de aprendizaje deben ser eso, una orientación hacia el estudiante y por ende, dar un giro al papel del profesor.

c) Las demandas crecientes de una sociedad de aprendizaje permanente y de una mayor flexibilidad en la organización del aprendizaje.

La flexibilidad en la Educación Superior ha sido un tema pendiente en el quehacer educativo, así como la integralidad, por ello las competencias buscan atender las demandas sociales en Educación Superior.

d) Se tienen en cuenta la búsqueda de mayores niveles de empleabilidad y de ciudadanía.

En la medida que los graduados de pregrado cuenten con los conocimientos, habilidades y valores que las empresas y las sociedades requieren, en esa medida los graduados podrán tener un empleo.

e) Un impulso a la dimensión europea de la Educación Superior.

Como consecuencia de este nuevo enfoque basado en competencias, tanto la educación europea en un principio, la Educación Superior en América Latina y el Caribe en una siguiente fase y la Educación Superior en Colombia se refrescan y dirigen de mejor manera sus esfuerzos los cuales son acordes a las características de los estudiantes de este siglo.

f) El suministro de un lenguaje más adecuado para el intercambio y el diálogo con los interesados.

A partir de este enfoque se han generalizado los conceptos utilizados, hoy en día no es raro entre los profesores hablar de competencias, competencias genéricas y específicas, de conocimientos, habilidades, valores y el nuevo rol del profesor. También es cierto que hay actividades pendientes para consolidar este nuevo paradigma, pero los avances han sido significativos en el ámbito europeo, América Latina y Colombia.

\subsubsection{Tipos de competencias}

Una vez definida la competencia y comentadas sus ventajas, es necesario que se indiquen los tipos de 
Ingenierías USBMed, Vol. 8, No. 1, Enero-Junio 2017

competencias que existe. Estas pueden ser genéricas o específicas, las genéricas identifican los elementos compartidos que deben ser comunes en todas las profesiones, mientras que las competencias específicas, son las que se tienen que desarrollar en un área temática específica [5].

Las competencias genéricas según González y Wagenaar [3] se dividen en:

- Instrumentales

- Personales y

- Sistemáticas.

A su vez las instrumentales Sierra Alonso [5] establece están divididas en capacidades metodológicas las cuales consideran las competencias:

- Capacidad de análisis y de síntesis.

- Capacidad de planificar y organizar.

- Resolución de problemas.

- Toma de decisiones.

El mismo autor enlista las habilidades cognitivas:

- Conocimientos generales básicos.

- Conocimientos básicos de la profesión.

Así como las destrezas tecnológicas:

- Habilidades básicas en el manejo de computadoras.

- Habilidades de la gestión de la información.

Y las destrezas lingüísticas:

- Comunicación oral y escrita en su propio idioma.

- Conocimientos de un segundo idioma.

Por lo que se refiere a las competencias genéricas personales, Sierra Alonso [5] indica que son:

- Capacidad crítica y autocrítica.

- Trabajo en equipo.

- Habilidades interpersonales.

- Capacidad de trabajar en equipo interdisciplinar.

- Capacidad de comunicarse con expertos de otras áreas.

- Apreciación de la diversidad y la multiculturalidad.

- Habilidad de trabajar en un contexto internacional.

- Compromiso ético.

Por último, Sierra Alonso [5] enlista las competencias genéricas sistemáticas que son:

- Capacidad de aplicar los conocimientos en la práctica.

- Habilidades de investigación.

- Capacidad de aprender.

- Capacidad de adaptarse a nuevas situaciones.

- Capacidad de generar nuevas ideas.

- Liderazgo.

- Conocimiento de cultura y costumbres de otros países.

- Habilidad para trabajar en forma autónoma.

- Diseño y gestión de proyectos.

- Iniciativa y espíritu emprendedor.
- Preocupación por la calidad.

- Motivación por el logro.

En cuanto a las competencias específicas no se profundiza debido a que cada programa educativo debe determinarlas.

\subsubsection{Competencias genéricas deseables}

La Agencia Nacional de Evaluación de la Calidad y Acreditación (ANECA) de España tiene como objetivo contribuir a la mejora de la calidad del sistema de Educación Superior, y realizó un estudio para determinar las competencias genéricas más apreciadas en la rama científica y tecnológica.

Dicho estudio determinó las 3 competencias genéricas más valoradas en cada una de las categorías, instrumentales, personales y sistemáticas. Las competencias que arrojó el estudio se muestran en orden de prioridad en la Tabla 1.

Tabla 1. Competencias genéricas más valoradas en los títulos de grado de la rama Científica Tecnológica [6]

\begin{tabular}{|c|c|}
\hline $\begin{array}{l}\text { Tipo de competencia } \\
\text { genérica }\end{array}$ & Competencia deseada \\
\hline \multirow[t]{3}{*}{ Instrumentales } & 1. Capacidad de análisis y síntesis. \\
\hline & 2. Resolución de problemas. \\
\hline & $\begin{array}{l}\text { Capacidad de organización y } \\
\text { planificación. }\end{array}$ \\
\hline \multirow[t]{3}{*}{ Personales } & 1. Compromiso ético. \\
\hline & 2. Trabajo en equipo. \\
\hline & 3. Capacidad crítica y autocrítica. \\
\hline \multirow[t]{3}{*}{ Sistemáticas } & $\begin{array}{l}\text { 1. Capacidad de adaptarse a } \\
\text { nuevas situaciones. }\end{array}$ \\
\hline & 2. Motivación por la calidad. \\
\hline & $\begin{array}{l}\text { 3. Capacidad de aprender o } \\
\text { aprendizaje autónomo. }\end{array}$ \\
\hline
\end{tabular}

Tipo de competencia genérica competencia deseada. Instrumentales

1. Capacidad de análisis y síntesis.

2. Resolución de problemas.

3. Capacidad de organización y planificación.

Personales

1. Compromiso ético.

2. Trabajo en equipo.

3. Capacidad crítica y autocrítica.

Sistemáticas

1. Capacidad de adaptarse a nuevas situaciones.

2. Motivación por la calidad.

3. Capacidad de aprender o aprendizaje autónomo.

Con esto queda muy claro cuáles deben ser las competencias que debe tener cualquier egresado de la universidad y desde cualquier modelo educativo se debe procurar generarlas. 
Ingenierías USBMed, Vol. 8, No. 1, Enero-Junio 2017

\subsubsection{Ingenieros y sus competencias específicas}

Hay autores [7] [8] [9] que desde hace muchos años han disertado sobre las competencias del ingeniero como consecuencia de la globalización, ellos han mencionado las siete tendencias que cambiaría la práctica de la ingeniería de manera significativa. Entre las tendencias se encuentran: la amplia gama de nuevos materiales y procesos que amplían el espacio de diseño de un ingeniero; El uso omnipresente de la tecnología de la información; El número creciente y la complejidad de las limitaciones (costos, seguridad, ecología); EI aumento de la necesidad de tener tanto conocimientos técnicos específicos como amplitud de conocimientos; La necesidad de trabajo en equipo y amplio conocimiento de negocios; El rápido ritmo de cambio que exige el aprendizaje permanente; La globalización, el cambio de una empresa de ingeniería nacional diferenciada a una que es mucho más cosmopolita. Para lo anterior han propuesto metodologías educativas para los ingenieros [10].

Existen trabajos más específicos indicando que los ingenieros deben tener la competencia de trabajo en equipo [11], otras investigaciones hablan sobre la competencia comunicativa en inglés [12], otros autores se enfocan a la estadística [13] y las matemáticas [14].

En general, son muchas las competencias específicas que debe poseer un ingeniero y son motivos de otra investigación, en este trabajo nos enfocaremos a las competencias genéricas y no a las específicas.

\subsection{Flipped Classroom}

En los últimos años se ha escrito mucho sobre el Flipped Classroom o aula volteada, pero no se han enfocado a la disciplina de la ingeniería, por ese motivo se ha escrito este trabajo.

Si iniciamos con la definición, autores como Lage, Platt y Treglia [15] definen Flipped Classroom de manera muy sencilla, indicando que las actividades que se realizan en el aula ahora se llevan a cabo fuera de ella y las que comúnmente se ejecutan fuera de ella, ahora son dentro.

En este trabajo, el Flipped Classroom en combinación con el Blended Learning, se considera como una estrategia pedagógica, la cual consiste en que el estudiante realice ahora en su casa las actividades que normalmente hace en el aula, como leer y realizar ejercicios, y en el aula aproveche la presencia del profesor para resolver dudas, debatir y reflexionar, siempre contando con conocimiento previo del tema a trabajar en el aula.

Flipped Classroom no es solo intercambiar las actividades entre el aula y la casa, es más que eso, es una estrategia que permite aprovechar la tecnología y el tiempo en casa, así como optimizar los tiempos en el aula aprovechando los conocimientos, habilidades y experiencias del profesor en el aula. Todo lo anterior con el apoyo de una plataforma educativa que sirva en primer término como un repositorio y de manera avanzada, crear un ambiente de aprendizaje basado en el concepto de micromundo de aprendizaje.

De esta manera las clases presenciales adquieren un mayor valor, el estudiante llega preparado para potenciar sus conocimientos y no a escuchar por primera vez el tema como sucede en una clase normal.

\subsubsection{Principios para el Flipped Classroom}

Una vez analizada la definición, es necesario mencionar los principios del Flipped Classroom. Kim y otros autores [16] han diseñado 9 principios para el éxito del Flipped Classroom los cuales son:

1. Proporcionar una oportunidad para que los estudiantes adquieran una primera explicación previa a la clase.

2. Dar un incentivo para que los estudiantes se preparen para la clase.

3. Facilitar un mecanismo para evaluar la comprensión del estudiante.

4. Proveer conexiones claras entre las actividades en clase y fuera de clase.

5. Proporcionar una orientación claramente definida y bien estructurada.

6. Otorgar suficiente tiempo a los estudiantes para llevar a cabo las tareas.

7. Dar las facilidades para la construcción de una comunidad de aprendizaje.

8. Aportar retroalimentación inmediata en trabajos individuales o grupales.

9. Equipar con tecnologías comunes y de fácil acceso.

Como se puede apreciar estos principios son básicos y fáciles de entender, su complejidad radica en cumplirlos al momento de aplicar Flipped Classroom.

\subsubsection{Recomendaciones para implementar Flipped Classroom}

Por otro lado, Rotellar y Cain [17] indican una serie de recomendaciones para implementar Flipped Classroom las cuales son:

1. Utilizar a los diseñadores instruccionales para determinar el contenido que se debe trabajar en casa y cómo se realizarán las actividades de aprendizaje en la clase.

2. Proporcionar numerosas oportunidades para desarrollar las habilidades individuales y grupales que permitan a los miembros de la facultad el cambio de paradigma.

3. Reconocer que el aumento de la dinámica en el aula puede resultar en la necesidad de personal adicional en el aula (por ejemplo, asistentes).

4. Reconocer que "cambiar las reglas" causará angustia entre muchos estudiantes que pueden incluso necesitar ser reeducados sobre cómo se tiene éxito en el aula invertida.

5. Vincular todas las actividades en casa (preclase) con las actividades en la clase, por lo que es necesario que los estudiantes cumplan con las actividades preclase, para tener éxito. 
Ingenierías USBMed, Vol. 8, No. 1, Enero-Junio 2017

6. Proporcionar orientación a los estudiantes para que puedan entender exactamente lo que necesitan saber o ser capaces de hacer cuando vienen a clase.

7. Proporcionar una oportunidad para que los estudiantes hagan preguntas y/o aclaren la información contenida en los materiales preclase.

8. Enfocarse en las actividades de la clase sobre cómo se puede agregar valor para ayudar a los estudiantes a pensar como expertos.

9. Proporcionar oportunidades de evaluación formativa para los estudiantes para entender lo que hacen y no saben.

10. Resistir la tentación de "volver a enseñar" el contenido a los estudiantes que no se preparan previamente. Ellos deben comprender lo que significa ser responsables y realizar las actividades preclase.

11. Tener cuidado de no sobrecargar a los estudiantes con mucho contenido preclase.

12. Evaluar los conocimientos, habilidades, comportamientos y actitudes que se desean de los estudiantes.

13. Estar preparado para ajustar las actividades de enseñanza y aprendizaje a medida que se descubre lo que contribuyó al aprendizaje y lo que no lo hizo.

Con los principios y siguiendo estas recomendaciones se puede tener mayores probabilidades de éxito al implantar el Flipped Classroom.

\subsubsection{Ventajas del Flipped Classroom}

Después de analizar el Flipped Classroom estamos en la posibilidad de enumerar las tres principales ventajas que apreciamos.

1. Mejor planeación. En las clases tradicionales el profesor puede llegar al aula sin una planeación de la clase, confiando en su experiencia y en sus conocimientos, por lo cual puede haber frases al inicio de la clase como "¿en qué nos quedamos la sesión anterior?". Dentro del Flipped Classroom esto no ocurre porque todas las sesiones presenciales deben estar planeadas desde el inicio del curso.

2. Mayor preparación del profesor. El Flipped Classroom exige una mayor expertiz del profesor, en el modelo actual un profesor que enseña estadística puede llegar al aula y escribir un ejercicio que ha aprendido de memoria, aunque realmente no domine el tema, el estudiante lo copia y se lo lleva a casa para entenderlo después. En el Flipped Classroom el estudiante intentó resolver el ejercicio en casa y si no lo logró trae dudas concretas para el profesor lo cual exige del profesor un mayor dominio del tema que enseña.

3. Desarrollo de las competencias de los estudiantes de pregrado. Este nuevo paradigma está diseñado para que el estudiante desarrolle las competencias genéricas, la mejor manera de desarrollar las competencias instrumentales, personales y sistemáticas es autoaprendiendo con la guía del profesor, de esa manera aprenderán a analizar, sintetizar, resolver problemas y demás competencias genéricas deseables.

Sin duda hay más ventajas al adoptar este paradigma, por el momento solo hemos mencionado las principales.

\section{Propuesta}

En este segundo apartado se explica la propuesta para implentar el Flipped Classroom en ingeniería, la cual puede ser una propuesta para aplicar en todos los pregrados. La propuesta de este trabajo se ve reflejada en la Figura 1.

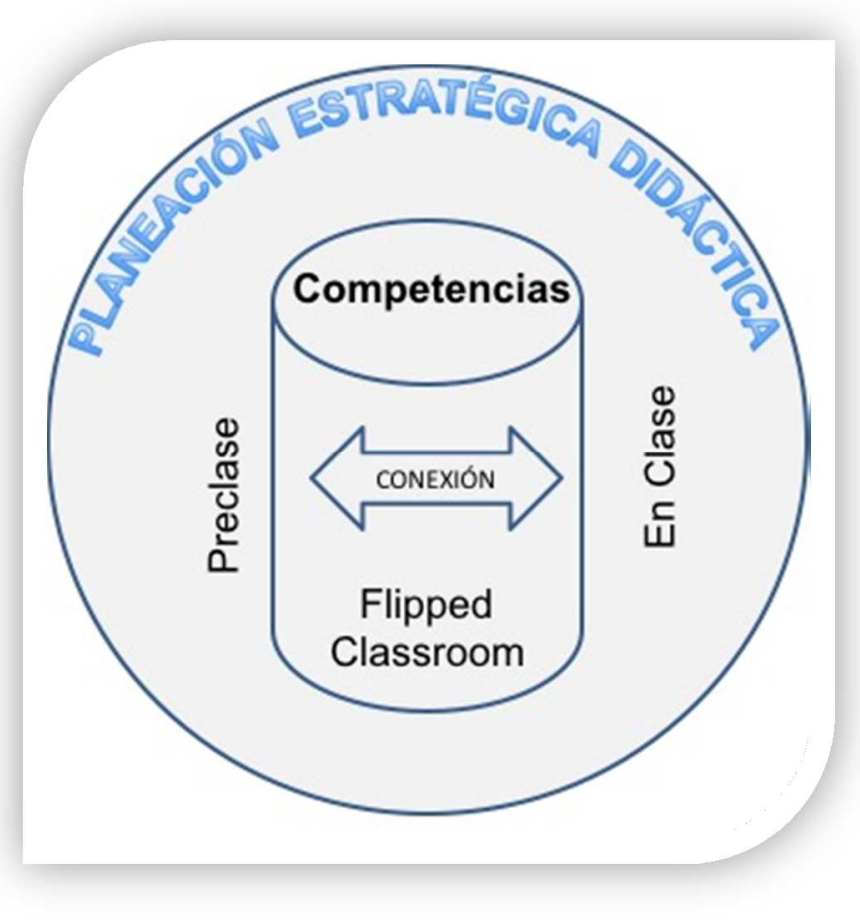

Figura 1. Flipped Classroom para desarrollar las competencias.

La propuesta muestra como el Flipped Classroom es la base para el desarrollo de las competencias, mientras que los dos pilares son las actividades preclase y las actividades en clase.

Las actividades preclase son estratégicamente diseñadas para que el estudiante desarrolle los conocimientos y habilidades previos a su clase. Mientras que las actividades en clase sirven para potenciar los conocimientos y habilidades previamente adquiridos, no es una repetición de lo tratado en la preclase, sino dudas específicas sobre lo teórico y la mejora de las habilidades para lo práctico.

Con la propuesta anterior, un estudiante no llegará a clase sin saber del tema a tratar, a menos que no cumpla con lo planeado.

Dentro del cilindro se encuentra la conexión que debe existir entre las actividades preclase y las actividades en clase, por ello el círculo que amalgama todo el proceso es la planeación estratégica didáctica. 
Si se logra diseñar adecuadamente una experiencia educativa bajo esta propuesta, se garantiza mayores probabilidades de éxito.

$\mathrm{Si}$ se recuerdan las competencias genéricas deseables tratadas previamente, dentro de las instrumentales están la capacidad de análisis y síntesis, resolución de problemas y la capacidad de organización y planificación. Por lo anterior surge una interrogante, ¿cómo desarrollar estas competencias dentro del aula? La respuesta es que en el aula es poco el tiempo y es muy difícil alcanzar la meta, sin embargo, mediante la propuesta de Flipped Classroom se pueden generar estas competencias en preclase y reforzarlas en la clase.

Así mismo, las competencias personales deseables son el compromiso ético, el trabajo en equipo y la tercera competencia es la capacidad crítica y autocrítica. Al igual que las competencias instrumentales estas competencias se desarrollan mejor mediante la propuesta Flipped Classroom, debido a que se requiere el compromiso ético, el trabajo en equipo preclase, la crítica y autocrítica para cumplir con las actividades propuestas.

Finalmente, si se habla sobre las competencias sistemáticas deseables, se puede decir que son las mejor orientadas a esta propuesta, la competencia de adaptarse a nuevas situaciones, conecta con la nueva forma de hacer las cosas y la capacidad de aprender y autoaprender son requisitos indispensables para Flipped Classroom y cada día que pasa en este modelo se desarrollan con mayor fuerza dichas competencias.

\section{Conclusiones}

Como conclusiones se tienen las siguientes.

1. Existen competencias genéricas que debería tener todo estudiante de ingeniería en pregrado y la forma de desarrollarlas puede variar.

2. El Flipped Classroom más que un modelo pedagógico, es un cambio de paradigma que permite aprovechar mejor las actividades preclase y las actividades en clase.

3. El Flipped Classroom es una estrategia idónea para desarrollar las competencias genéricas en estudiantes de pregrado.
4. No hay modelo pedagógico o propuesta didáctica que tenga éxito sin el trabajo arduo del profesor.

5. Efectivamente el profesor necesita mejor planeación y mayor expertiz si desea cambiar de la forma tradicional de trabajar y ahora hacerlo mediante el Flipped Classroom.

\section{Referencias}

[1] RAE, «Diccionario de la lengua española,» 2016. [En línea]. Available: http://dle.rae.es/?id=A0fanvT|A0gTnnL. [Último acceso: 30 Junio 2016].

[2] C. J. Van-der Hofstadt Román y J. M. Gómez Gras, Competencias y habilidades profesionales para universitarios, Madrid: Diaz de Santos, 2006, p. 505.

[3] J. González y R. Wagenaar, Tuning Educational Structures in Europe Informe Final. Fase Uno, Universidad de Deusto, 2003.

[4] OCDE, Defining and Selecting Key Competencies, 2001, p. 264.

[5] M. I. Sierra-Alonso, Desarrollo - Evaluación de competencias genéricas en los estudiantes universitarios, Madrid: Dykinson, 2011, p. 154.

[6] M. I. Sierra Alonso, Desarrollo - Evaluación de competencias genéricas en los estudiantes universitarios, Madrid: Dykinson, 2011, p. 154.

[7] W. Wulf, «Changing nature of engineering,» 1997. [En línea]. Available: http://www.nae.edu/nae/ bridgecom.nsf/weblinks/NAEW4NHMBD?OpenDocument. [Último acceso: 2015].

[8] J. R. Lohmann, H. A. Rollins y J. Hoey, «Defining, developing and assessing global competence in engineers,» European Journal of Engineering Education, vol. 31, n⿳0 1, p. 119-131, 2006.

[9] W. Ravesteijn, E. De Graaff y O. Kroesen, «Engineering the future: the social necessity of communicative engineers,» European Journal of Engineering Education, vol. 31, $\mathrm{n}^{\circ}$ 1, p. 63-71, 2006.

[10] J. E. Parra Castrillón, M. J. Amariles Camacho y C. A. Castro Castro, «Aprendizaje basado en problemas en el camino a la innovación en ingeniería,» Ingenierías USBMed, vol. 7, nº 2, pp. 96-103, 2016.

[11] E. G. Adams, «Building Successful Student Teams in the Engineering Classroom,» Journal of STEM Education, vol. 4, n 3, pp. 1-6, 2003.

[12] L. M. Barreiro-Perez y M. d. C. Batista-Gonzalez, «El desarrollo de la competencia comunicativa en inglés ingenieros y arquitectos,» Arquitectura y urbanismo, vol. XXXVII, no 1, pp. 97-102, 2016.

[13] S. M. Ramírez-Buelvas, L. R. Cid-Serrano, H. Castillo-Sánchez, M. d. P. Marín-Gaviria, D. González-Gómez y R. Medina-Muñoz, «Implementación y evaluación de un curso de estadística basado en competencias,» Scientia et Technica, vol. XVI, n 49, pp. 392-401, 2011.

[14] A. Vintere y A. Zeidmane, «Research in Mathematical competence in Engineers' professional activities,» Engineerring for rural development , pp. 497-504, 2014.

[15] M. Lage, G. Platt y M. Treglia, «Inverting the Classroom: A Gateway to Creating an Inclusive Learning Environment,» The Journal of Economic Education, vol. 31, nº 1, pp. 30-43, 2000.

[16] M. K. Kim, S. M. Kim, O. Khera y J. Getman, «The experience of three flipped classrooms in an urban university: an exploration of design principles,» Internet and Higher Education, vol. 22, pp. 37-50, 2014.

[17] C. Rotellar y J. Cain, «Research, Perspectives, and Recommendations on Implementing the Flipped Classroom,» American Journal of Pharmaceutical Education, vol. 80, nº 2, pp. 1-10, 2016 